\title{
Suplementación de cobayos (Cavia porcellus L.) con follajes fresco de morera (Morus alba) y moringa (Mo- ringa oleifera)
}

\section{Guinea pigs (Cavia porcellus L.) suplementation with fresh foliage of morera (Morus alba) and moringa (Moringa oleifera)}

\section{Nadir Reyes-Sánchez ${ }^{1}$, Jerry Vivas ${ }^{2}$, Jorge Aguilar ${ }^{3}$, Janin Hernández ${ }^{3}$, Norlan Caldera ${ }^{4}$}

${ }^{1} \mathrm{PhD}$ en ciencia animal, ORCID: https://orcid.org/0000-0002-5579-9396 / 2 MSc. en agroecología y desarrollo sostenible, ORCID: https:// orcid.org/0000-0001-9681-7215 / ${ }^{3}$ Docentes investigadores / ${ }^{4}$ MSc. en producción animal, ORCID: https://orcid.org/0000-0002-0807-7921 Autor para correspondencia: nadir.reyes@ci.una.edu.ni Universidad Nacional Agraria.

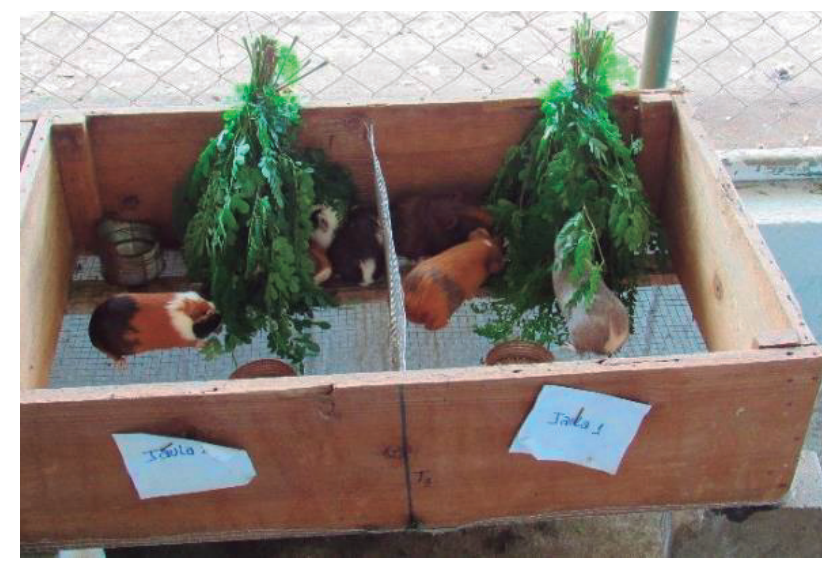

\section{RESUMEN}

Se realizó este experimento para evaluar la suplementación alimenticia para cobayos (Cavia porcellus L.) con follaje fresco de Moringa oleifera y Morus alba (Morera) y su efecto sobre el comportamiento productivo. Se utilizaron 18 cobayos mestizos de 30 días de edad, con un peso vivo inicial promedio de 175.8 gramos. Se distribuyeron en un diseño completamente al azar en tres tratamientos, con seis repeticiones. Los tratamientos evaluados fueron tres: 1) concentrado comercial (CC) a voluntad + follaje fresco de CT-115, 2) $70 \%$ CC + follaje fresco de Morera y 3) $70 \% \mathrm{CC}+$ follaje fresco de Moringa. Los resultados indican que la alimentación de cobayos con $70 \%$ de CC y suplementados con follaje fresco de Morera o Moringa no afectan significativamente el comportamiento productivo (peso vivo, ganancia de peso y conversión alimenticia), en comparación con cobayos alimentados con CC a voluntad + CT115. La alimentación de cobayos con $70 \%$ de $\mathrm{CC}+$ follaje fresco de Moringa o 70\% de CC + follaje fresco de Morera, al reducir los costos de producción, generan mayor beneficio financiero que utilizar CC a voluntad + CT-115, siendo la ración 70\% de CC + follaje fresco de Moringa la que genera mayor ventaja financiera.

Palabras clave: comportamiento productivo, peso vivo, ganancia de peso, conversión alimenticia.
A research study was conducted to evaluate guinea pig (Cavia porcellus L.) supplementation with Moringa oleifera fresh foliage or Morus alba fresh foliage and the effect on productive behavior. A total of 18 creole guinea pigs with initial body weight of $175.8 \mathrm{~g}$ were used in a Completely Randomized Design and with three treatments: 1) Commercial concentrate (CC) ad libitum + CT-115, 2) $70 \% \mathrm{CC}+$ Moringa oleifera fresh foliage and 3) 70\% CC + Morus alba fresh foliage with six repetitions. No significant effects $(\mathrm{P}>0.05 \%)$ were observed between T1, T2 and T3 related with productive parameters (Body weight, gain weight and feed conversion ratio). Guinea pigs feeding with $70 \%$ CC + Moringa oleifera fresh foliage or $70 \% \mathrm{CC}$ + Morus alba fresh foliage reduced cost production and increase financial benefit compared with guinea pigs feeding with $\mathrm{CC}$ ad libitum + CT-115. However, T3: 70\% CC + Moringa oleifera fresh foliage showed the best financial results.

Keys words: productive behavior, live weight, weight gain, feed conversion
Recibido: 5 de marzo del 2018 Aceptado: 29 de mayo del 2018
Los artículos de la revista La Calera de la Universidad Nacional Agraria, Nicaragua, se comparten bajo términos de la licencia Creative Commons: Reconocimiento, No Comercial, Compartir Igual. Las autorizaciones adicionales a las aquí delimitadas se pueden obtener en el correo freddy.aleman@ci.una.edu.ni 


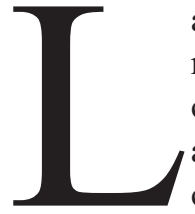

a capacidad futura de la humanidad para alimentarse está en peligro a causa de la creciente presión sobre los recursos naturales, el aumento de la desigualdad y los efectos del cambio climático. El planeta está al límite de la sostenibilidad en términos de producción de alimentos, tenemos que encontrar nuevas fuentes alternativas de alimentación (FAO, 2017).

El cobayo es una alternativa sostenible, respetuosa con el ambiente y presente en la mesa latinoamericana desde tiempos ancestrales, tanto por costumbre, como por su alto contenido nutricional. El cobayo es un pequeño roedor originario de los Andes, utilizado como alimento en una extensa región comprendida por Chile, Argentina, Bolivia, Perú, Ecuador y Colombia. Es un herbívoro monogástrico, que realiza una digestión enzimática del alimento en el estómago y una fermentación microbiana en el ciego, con un buen aprovechamiento de la fibra, realiza la cecotrófia para reutilizar y aprovechar los nutrientes generados en la fermentación cecal, lo que permite un buen comportamiento productivo con raciones a base de follaje.

La nutrición y alimentación juega un papel preponderante para mejorar su comportamiento productivo. Generalmente, la alimentación de cuyes requiere de alimentos comerciales para cubrir los requerimientos nutricionales. Esto representa aproximadamente $70 \%$ de los costos totales de producción y constituye la principal limitación para el productor. El follaje de numerosas especies de árboles y arbustos tropicales puede mejorar la calidad de las dietas tradicionalmente usadas en animales, por su alta capacidad de producción de biomasa y elevado contenido de proteína bruta (CATIE, 1985). Entre las especies arbustivas de interés para alimentación de cobayos se encuentra la Moringa y la Morera

Moringa oleifera es un árbol forrajero que crece bien en todo tipo de suelos desde ácidos hasta alcalinos, es tolerante a la sequía y con alta producción de forraje entre 24 y 99 ton MS/ha/año (Reyes-Sánchez et al., 2006), las hojas frescas contienen entre 17 y $24.6 \%$ de PB y 2.73 Mcal de EM/kg MS (Mendieta-Araica et al., 2009), es rico en vitaminas A, B y C, calcio, hierro y en dos aminoácidos esenciales (metionina y cistina) generalmente deficientes en otros alimentos (Makkar y Becker, 1996) y ha sido utilizado en una gran variedad de dietas para distintas especies animales (Reyes-Sánchez et al., 2006; Reyes-Sánchez et al., 2009; Mendieta-Araica et al., 2009; Mendieta-Araica et al., 2011; Rodríguez-Pérez et al., 2012).

El follaje de Morus alba contiene entre 15 y $28 \%$ de proteína bruta, depende de la variedad, edad y condiciones de crecimiento, y presenta más de $46 \%$ de aminoá- cidos esenciales (Machii, 1989). Las fracciones fibrosas son bajas comparadas con otros follajes (Shayo, 1997) y tiene alto contenido de minerales (hasta 17\% de cenizas), con valores elevados de calcio y fósforo (Sánchez, 1999).

El objetivo de este estudio fue evaluar el comportamiento productivo (consumo de alimento, peso vivo, ganancia de peso y conversión alimenticia) de cobayos suplementados con follaje fresco de Morus alba y Moringa oleifera.

\section{MATERIALES Y MÉTODOS}

Localización del experimento. El estudio se realizó en la granja de cobayos de la Facultad de Ciencia Animal de la Universidad Nacional Agraria (UNA) Managua, Nicaragua, localizada geográficamente e las coordenadas $12^{\circ} 8^{\prime} 33^{\prime \prime}$ de latitud norte y $86^{\circ} 10^{\prime} 31$ " de longitud oeste, a una altitud de $56 \mathrm{msnm}$, con temperatura media anual de $27.3{ }^{\circ} \mathrm{C}$, precipitación media anual de $1264.2 \mathrm{~mm}$ y humedad relativa del 73.2\% (INETER, 2009).

Diseño experimental y análisis estadístico. Se utilizaron 18 cobayos mestizos de 30 días de edad, con un peso vivo inicial promedio de 175.8 gramos, que se distribuyeron en un diseño completamente al azar con tres tratamientos, y seis repeticiones. Los tratamientos corresponden a: 1) concentrado comercial (CC) a voluntad + follaje fresco de CT-115, 2) 70\% CC + follaje fresco de Morera y 3) 70\% $\mathrm{CC}+$ follaje fresco de Moringa.

Los datos fueron sometidos al análisis de varianza, para determinar el efecto de la suplementación con Morera y Moringa sobre las variables en estudio, utilizando el General Lineal Model del Software Minitab versión 14.0 para computadoras personales (Minitab, 1998), y cuando se encontraron diferencias estadísticas significativas se realizó la prueba de Tukey $(p<0.05)$.

Manejo y alimentación de los animales. Los cuyes fueron alojados en jaulas de madera y malla metálica, provistas de comedero y bebedero. Fueron sometidos a un periodo de adaptación a los tratamientos, por ocho días; el cambio de ración se realizó gradualmente, se fue disminuyendo la cantidad de concentrado e incrementando la oferta de follaje de Morera y Moringa diariamente, hasta sustituir el forraje de CT-115. Además, los animales fueron vitaminados y desparasitados.

El concentrado comercial se suministró por la mañana (7:00 am). El forraje de CT-115 y el follaje de Morera y Moringa se obtuvieron de parcelas ubicadas contiguas a la Granja con edad de rebrote de 60 días, era cosechado diariamente con machete y ofertado dos veces 
al día (8:00 am y 1:00 pm), para garantizar follaje fresco. El Material ofrecido de Morera y Moringa consistía en follaje y peciolos no mayores a los $5 \mathrm{~mm}$ de diámetro. Los animales tuvieron acceso al agua ad libitum.

Finalizado el período de adaptación, los animales se pesaron e inició la fase experimental con una duración de nueve semanas. Cada componente de la ración se suministró de acuerdo con las proporciones establecidas por tratamiento con ajustes semanales.

El concentrado comercial utilizado fue un alimento balanceado de la empresa Cargill de Nicaragua, constituido de los siguientes ingredientes: maíz, sorgo, harina de soya, subproducto de arroz, subproducto de galleta, aromatizante (cítrico y manzana), carbonato de calcio, fosfato de calcio, cloruro de sodio, lisina, metionina, treonina, $\mathrm{Fe}, \mathrm{Cu}, \mathrm{Zn}, \mathrm{Mn}$, Se, I, Vitaminas $\mathrm{A}, \mathrm{D}_{3}, \mathrm{E}, \mathrm{K}_{3}, \mathrm{~B}_{1}$, $\mathrm{B}_{2}, \mathrm{~B}_{6}, \mathrm{~B}_{12}$, niacina, ácido pantoténico, ácido ascórbico, biotina, ácido fólico y aluminosilicatos de sodio y calcio como secuestrante de micotoxina.

Los animales se pesaban cada siete días, antes de suministrarles alimento, para determinar el peso vivo y calcular la ganancia de peso. El consumo voluntario de los alimentos fue estimado diariamente durante todo el período experimental por el método convencional, calculando la diferencia entre el alimento ofrecido y el alimentado rechazado. Por ello, los alimentos se pesaban antes de suministrárselos a los animales y al día siguiente antes de ofrecer el nuevo alimento, el alimento rechazado era colectado y pesado por separado.

Cuadro 1. Composición química de los alimentos

\begin{tabular}{lccccc}
\hline \multirow{2}{*}{ Follaje } & \multicolumn{5}{c}{ Composición química } \\
\cline { 2 - 6 } & MS (\%) & PB (\%) & FB (\%) & DIVMS (\%) & $\begin{array}{c}\text { ED (kcal/ } \\
\mathrm{kg})\end{array}$ \\
\hline CC * & 87.00 & 17.0 & 10.0 & - & 3800 \\
CT-115 & 19.50 & 12.0 & 23.9 & 58.3 & - \\
Morera & 18.85 & 23.0 & 19.5 & 66.4 & - \\
Moringa & 19.25 & 24.5 & 23.6 & 65.9 & - \\
\hline
\end{tabular}

* Análisis garantizado por la empresa Cargill.

El pasto CT-115 y el follaje de Morera y Moringa ofrecido se muestreó cada 15 días y se almacenaron en un congelador. Las muestras posteriormente fueron secadas en un horno de circulación forzada de aire a $65^{\circ} \mathrm{C}$ por 48 horas, luego se molieron hasta obtener un tamaño de partícula de un milímetro, y se almacenaron en recipientes de vidrios debidamente identificados, para subsecuentes análisis químicos.
Análisis químicos. A las muestras de pasto CT-115, Morera y Moringa se les determinó el contenido de materia seca (MS), proteína bruta (PB) y la digestibilidad in vitro de la materia seca (DIVMS). La MS fue determinada por secado de la muestra en un horno a $105^{\circ} \mathrm{C}$ durante seis horas según el procedimiento de la AOAC (1990). La concentración de nitrógeno total fue determinada utilizando el método de Kjeldahl (AOAC, 1984) y el contenido de proteína bruta fue calculado mediante la fórmula $\mathrm{PB}=\%$ de nitrógeno total * 6.25 (factor de ajuste de la proteína vegetal).

\section{RESULTADOS Y DISCUSIÓN}

La composición química de los alimentos utilizados se presenta en el cuadro 1. El concentrado comercial (CC) es un alimento de poco volumen, con elevada concentración de nutrientes, alta digestibilidad y bajo contenido de fibras. La síntesis o formación de tejido corporal requiere del aporte de proteínas. El contenido de PB del CC (17\%), es similar al requerimiento de $18 \%$ de $\mathrm{PB}$ recomendado por NRC (1995) para cobayos en crecimiento.

El contenido de energía del CC (3 $800 \mathrm{kcal} / \mathrm{kg}$ ) es superior a las $3000 \mathrm{kcal} / \mathrm{kg}$ recomendado por la NRC (1995), no obstante, diversos autores (Mercado, 1972; Paucar, 1992; Torres et al., 2006) coinciden en que el nivel de energía ofrecido en la dieta es directamente proporcional a la respuesta animal en ganancia de peso. Al respecto, Chauca de Zaldivar (1997) sugiere que los cobayos alimentados con dietas de mayor densidad energética tienen una mejor respuesta en términos de ganancia de peso y eficiencia alimenticia.

La concentración de fibra del CC es del $10 \%$ (cuadro 1), menor $15 \%$ recomendado por la NRC (1995) para alimentación de cobayos. Sin embargo, es importante destacar que el suministro de fibra del CC pierde importancia cuando los animales reciben una alimentación mixta (concentrado más forraje), ya que el aporte de fibra está dado básicamente por el consumo de los forrajes.

El valor nutritivo de las plantas forrajeras está en función de la composición química y del consumo voluntario de los animales. El forraje asegura la ingestión adecuada de fibra y ayuda a cubrir, en parte, los requerimientos de algunos nutrientes. El alimento concentrado satisface los requerimientos de proteína, energía, minerales y vitaminas. Los contenidos de MS (\%), PB (\%), FB (\%) y DIVMS (\%) del CT-115, Morera y Moringa se presentan en el cuadro 1. La concentración de PB (\%) del follaje de Morera y Moringa es superior al requerimiento de PB de los cobayos en crecimiento (17\%), según lo re- 
comendado por la NRC (1995), lo que las convierte en un alimento forrajero de excelente calidad para los cobayos.

El contenido de fibra de la dieta tiene importancia en la composición de las raciones no solo por la capacidad que tienen los cobayos de digerirla, sino que su inclusión es necesaria para favorecer la digestibilidad de otros nutrientes, ya que retarda el pasaje del contenido alimenticio a través de tracto digestivo. En ese sentido, el aporte de fibra de la Morera y la Moringa (19.5 y 23.6\%, respectivamente), complementa adecuadamente las necesidades de FB de los cobayos.

Según Moreno (1989); Cairampoma et al., (1991); Villafranca (2003); Ciprian (2005), un alimento concentrado para cobayos en crecimiento, debe proporcionar un mínimo de $17 \%$ de proteína, $2.9 \mathrm{Mcal}$ ED/kg MS y un rango de 8 a 14\% de fibra cruda, valores similares a los del CC utilizado en este estudio.

Peso vivo y ganancia de peso. El aumento de peso vivo es la respuesta de los animales ante el consumo de una dieta y refleja directamente que mientras mayor sea la cantidad
El tamaño y velocidad de crecimiento de los cobayos, está influenciada por la alimentación y el genotipo del individuo. Los cobayos mejorados alcanzan incrementos diarios de peso de $15.4 \mathrm{~g}$ (Saravia, 1994); los cruzados ganan 8-10 g diarios (Chauca, 1995) y los cobayos criollos obtienen un incremento diario aproximado de 3.2 a 4 g (Higaona et al., 1990; Chauca, 1995). La ganancia media diaria de los cobayos (6.42-6.84) que consumieron las dietas experimentales (cuadro 2) está dentro del rango reportados por estos autores, para este genotipo.

La ganancia diaria de peso de los cobayos registrada es similar a la reportadas por Miranda (2014) y Paredes (1972) que obtuvieron entre 6.41 y $7.61 \mathrm{~g} / \mathrm{d}$ y entre 7.9 y $8.36 \mathrm{~g}$, en cobayos alimentados con forraje hidropónico de cebada y con alfalfa más alimento concentrado, respectivamente.

En la figura 1 se puede observar que los pesos iniciales entre tratamientos no muestran diferencia significativa $(p>0.05)$. No se encontró diferencia estadística significativa $(p>0.05)$ en los pesos vivos durante todo el periodo experimental.

Cuadro 2. Peso vivo, ganancia de peso total y ganancia media diaria de cobayos suplementados con follaje de Morera y Moringa

\begin{tabular}{lcccc}
\hline \multicolumn{1}{c}{ Tratamientos } & $\begin{array}{c}\text { Peso vivo } \\
\text { inicial }(\mathrm{g})\end{array}$ & $\begin{array}{c}\text { Peso vivo } \\
\text { final }(\mathrm{g})\end{array}$ & $\begin{array}{c}\text { Ganancia } \\
\text { peso total }(\mathrm{g})\end{array}$ & $\begin{array}{c}\text { Ganancia } \\
\text { media diaria } \\
(\mathrm{g} / \mathrm{d})\end{array}$ \\
\hline CC a voluntad + CT-115 & 176.3 & 607.0 & 430.7 & 6.84 \\
$70 \%$ CC + Morera & 176.3 & 580.8 & 404.5 & 6.42 \\
$70 \%$ CC + Moringa & 174.7 & 590.2 & 415.5 & 6.60 \\
\hline
\end{tabular}

Los cobayos alimentados con CC a voluntad + follaje fresco de CT-115 tuvieron similar comportamiento del peso vivo, que los cobayos alimentados con $70 \% \mathrm{CC}$ + follaje fresco de Morera y los alimentados con $70 \% \mathrm{CC}+$ follaje fresco de Moringa.

de nutrientes que un animal tenga disponible, siempre y cuando los digiera y absorba eficientemente, mayor será la magnitud del peso que demuestre.

Con relación al peso vivo final, ganancia de peso total y ganancia media diaria de los cobayos, no se observaron diferencias significativas $(p>0.05)$ entre las dietas experimentales estudiadas (cuadro 2). Encontrar similares respuestas productivas $(p>0.05)$, indica que el contenido de nutrientes de los follajes suplementarios de Morera y Moringa balancean la ración, alcanzando niveles de respuesta comparables al del CC a voluntad + CT-115. Al respecto, Ccahuana (2008), Inga (2008), Villafranca (2003) tampoco encontraron diferencias en la ganancia de peso diaria entre cobayos al evaluar diferentes tipos de dietas.

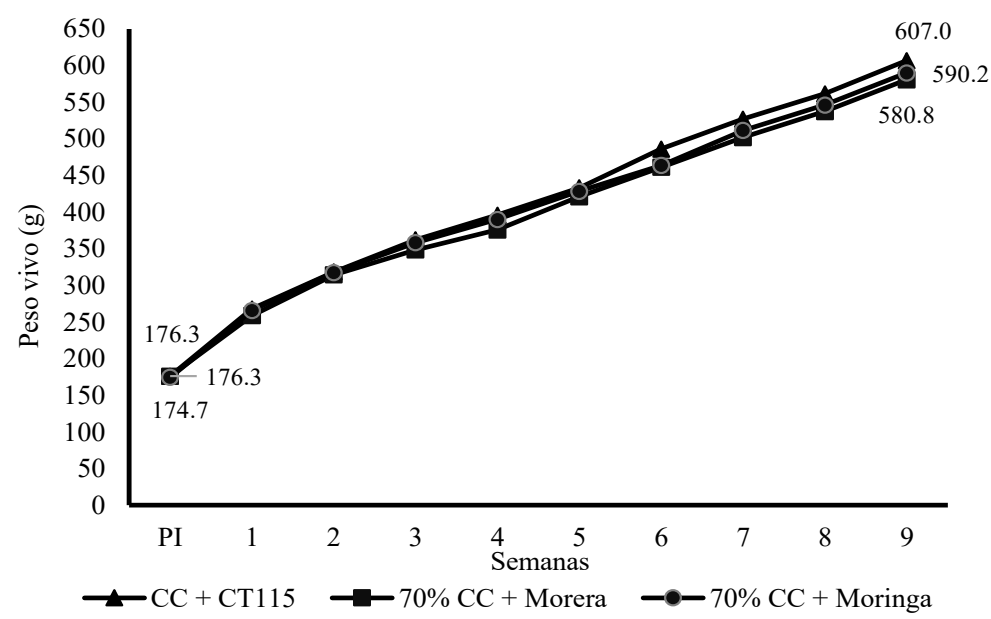

Figura 1. Curva de crecimiento (peso vivo en gramos) de cobayos suplementados con follaje de Morera y Moringa. 
Consumo de alimento (MS). El consumo de MS de CC, consumo de MS de forraje, consumo total de MS y consumo acumulado de CC durante el período experimental, fueron estadísticamente superiores $(p<0.05)$ en los cobayos alimentados con CC a voluntad + follaje fresco de CT-115 que en los cobayos alimentados con $70 \%$ CC + follaje fresco de Morera o con $70 \%$ de CC + follaje fresco de Moringa, los que no difieren estadísticamente entre sí (cuadro 3 y figura 2).

Cuadro 3. Consumo de alimento y conversión alimenticia de cobayos suplementados con follaje de Morera y Moringa

\begin{tabular}{lccccc}
\hline \multicolumn{1}{c}{ Tratamientos } & $\begin{array}{c}\text { Consumo MS } \\
\text { CC }(\mathrm{g})\end{array}$ & $\begin{array}{c}\text { Consumo MS } \\
\text { Forraje }(\mathrm{g})\end{array}$ & $\begin{array}{c}\text { Consumo total } \\
\text { MS }(\mathrm{g})\end{array}$ & $\begin{array}{c}\text { Ganancia } \\
\text { peso }\end{array}$ & $\begin{array}{c}\text { Conversión } \\
\text { alimenticia }\end{array}$ \\
\hline CC a voluntad + CT-115 & $18.40 \mathrm{a}$ & $21.16 \mathrm{a}$ & $39.56 \mathrm{a}$ & $6.84 \mathrm{a}$ & $5.78 \mathrm{a}$ \\
$70 \% \mathrm{CC}+$ Morera & $12.60 \mathrm{~b}$ & $14.89 \mathrm{~b}$ & $27.49 \mathrm{~b}$ & $6.42 \mathrm{a}$ & $4.28 \mathrm{~b}$ \\
$70 \% \mathrm{CC}+$ Moringa & $11.33 \mathrm{c}$ & $15.92 \mathrm{~b}$ & $4.13 \mathrm{~b}$ & $27.25 \mathrm{~b}$ & $4.13 \mathrm{~b}$ \\
\hline
\end{tabular}

Valores en la columna seguidos de diferentes letras, difieren estadísticamente entre sí, Tuckey $(\mathrm{p}<0.05)$.

Las diferencias en consumo de MS de CC, era de esperarse, ya que un tratamiento tenía acceso a voluntad al concentrado comercial y los cobayos de los otros tratamientos tenían acceso restringido $(70 \% \mathrm{CC})$. No obstante, el consumo de MS de forraje y consumo total de MS estadísticamente superior $(p<0.05)$ en los cobayos alimentados con CC a voluntad + follaje fresco de CT-115, podría deberse a que el cobayo regula voluntariamente el consumo de alimento en función al contenido energético (Mc Donald et al., 2010).

El procesamiento de la fibra se da por fermentación microbiana a nivel del ciego y colon obteniendo entre sus productos ácidos grasos de cadena corta que contribuyen a satisfacer los requerimientos de energía de esta especie. Esto significa que al tener los follajes de Morera y Moringa mejor valor nutritivo (23 y $24.5 \%$ de PB y 66.4 y $65.9 \%$ DIVMS, respectivamente) que el follaje de CT-115 (12\% PB y $58.3 \%$ DIVMS) (cuadro 1), habría mayor disponibilidad de energía para cubrir sus necesidades energéticas lo que permite al cobayo regular el consumo voluntario en función de la cantidad de nutrientes disponibles para satisfacer sus requerimientos y por lo tanto necesita menor consumo total de MS (cuadro 3 ).

Similar resultado reporta Airahuacho (2007), que encontró diferencias significativas en el consumo de materia seca de los cobayos en función del contenido energético de las raciones experimentales.
Conversión alimenticia. El índice de conversión alimenticia indirectamente evalúa el uso de las raciones alimenticias en base a su digestibilidad, absorción y calidad de nutrientes (Mattos et al., 2003). En el caso particular de este estudio, la utilización de follaje de Morera y Moringa en las dietas experimentales es con el fin de encontrar una ración que disminuya la utilización de $\mathrm{CC}$ y obtener una conversión alimenticia que se aproxime a los valores de un alimento balanceado integral.

Correspondiendo a los resultados de consumo y ganancia de peso, las raciones $70 \% \mathrm{CC}+$ follaje de Morera y 70\% CC + follaje de Moringa, presentaron significativamente $(p<0.05)$ una mejor conversión alimenticia, con 4.28 y 4.13 respectivamente, que la ración de CC a voluntad + forraje de CT-169 con 5.78 (cuadro 3).

Esto indicaría que los cobayos alimentados con las raciones experimentales de 70\% CC + follaje de Morera y $70 \% \mathrm{CC}+$ follaje de Moringa mostraron una mayor eficiencia en la transformación del alimento en tejido corporal, alcanzando el peso comercial con un menor consumo de alimento. Similar resultado reporta Airahuacho (2007) que encontró diferencias significativas en cobayos que recibieron alimentos con diferente densidad energética.

Análisis financiero. Los resultados del análisis financiero demuestran que la alimentación de cobayos con $70 \%$ de

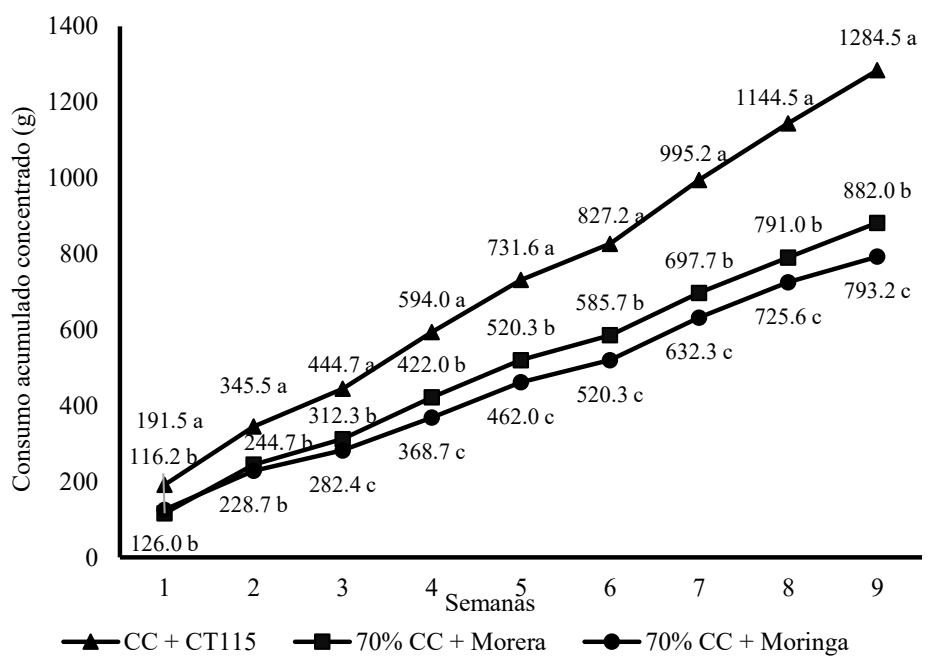

Figura 2. Consumo acumulado de concentrado comercial de cobayos suplementados con follaje de fresco de Morus alba y Moringa oleifera. 
Cuadro 4. Costo de producción de cobayos suplementados con follaje fresco de Moringa oleifera y follaje fresco de Morus alba

\begin{tabular}{lccccc}
\hline \multicolumn{1}{c}{ Tratamientos } & $\begin{array}{c}\text { Consumo } \\
\text { total CC }(\mathrm{kg})\end{array}$ & $\begin{array}{c}\text { Precio USD } \\
\text { CC }(\mathrm{kg})\end{array}$ & $\begin{array}{c}\text { Costo consumo } \\
\text { CC USD } \\
\mathrm{x} \mathrm{2)}\end{array}$ & $\begin{array}{c}\text { Peso vivo } \\
\text { final }\end{array}$ & $\begin{array}{c}\text { Costo pro- } \\
\text { ducción kg de } \\
\text { cobayo }\end{array}$ \\
\hline CC a voluntad + CT-115 & 1.285 & 0.48 & 0.62 & 0.607 & 1.02 \\
$70 \%$ CC + Morera & 0.882 & 0.48 & 0.42 & 0.509 & 0.83 \\
$70 \%$ CC + Moringa & 0.793 & 0.48 & 0.38 & 0.509 & 0.64 \\
\hline
\end{tabular}

$\mathrm{CC}+$ follaje fresco de Moringa genera los menores costos de producción (cuadro 4) de un kilogramo de cobayo en pie (USD 0.64) que los tratamientos con $70 \% \mathrm{CC}+$ follaje fresco de Morera (USD 0.83) y CC a voluntad + CT-115 (USD 1.03).

\section{CONCLUSIONES}

La alimentación de cobayos con $70 \%$ de $\mathrm{CC}$ y suplementados con follaje fresco de Morus alba o Moringa oleifera no afectan el comportamiento productivo (peso vivo, ganancia de peso y conversión alimenticia), en comparación con cobayos alimentados con $\mathrm{CC}$ a voluntad + CT-115. La alimentación de cobayos con $70 \%$ de $\mathrm{CC}+$ follaje fresco de Moringa oleifera o $70 \%$ de CC + follaje fresco de Morus alba al reducir los costos de producción, generan mayor beneficio financiero que utilizar CC a voluntad + CT-115, siendo la ración $70 \%$ de CC + follaje fresco de Moringa la que genera mayor ventaja financiera.

\section{REFERENCIAS BIBLIOGRÁFICAS}

AOAC (Association of official analytical chemists). 1984. Official methods of analysis. 14 ed. Washington DC, US. 1990. Official Methods of Analysis. 12 ed. Washington DC, US. 1018 p.

Airahuacho, B. 2007. Evaluación de dos niveles de energía digestible en base a los estándares nutricionales del NRC (1995) en dietas de crecimiento para cuyes (Cavia porcellus). Tesis Magíster. Lima, PE. Universidad Nacional Agraria La Molina. 85 p.

Cairampoma, V; Castro J, Chirinos, D. 1991. Adición de enzimas digestivas a suplementos con diferentes niveles de fibra en el engorde de cuyes. In XIV Reunión APPA. Cerro de Pasco: Asociación Peruana de Producción Animal.

CATIE (Centro Tropical de Investigación y Enseñanza, CR). 1985. Programa regional de capacitación para el desarrollo agrícola y la alimentación en el Istmo Centroamericano y la República Dominicana. In Políticas de inves tigación y desarrollo agropecuario. Memorias. Turrialba, CR. p 74-76.

Ccahuana L. 2008. Evaluación del bagazo de marigold en dietas peletizadas con exclusión de forraje verde para cuyes (Cavia porcellus) en crecimiento. Tesis Ing. Zootecnista. Lima, PE. Universidad Nacional Agraria

Chauca, D. 1995. Fisiología digestiva: Crianza de cuyes. Lima: INIA. Serie Guía Didáctica. p 13-16.

Chauca de Zaldívar, L. 1997. Producción de cuyes (Cavia porcellus). (en línea). Disponible en: http://www.fao.org/ docrep/W6562S/ W6562S00.htm

Ciprian, R. 2005. Evaluación del tamaño de partícula y nivel de fibra en el concentrado para cuyes (Cavia porcellus L.) en crecimiento. Tesis Magister Scientiae. Lima, PE. Universidad Nacional Agraria. La Molina. 74 p.

FAO. 2017. The future of food and agriculture - Trends and challenges. Rome. (en línea). Disponible en: http://www.fao.org/3/ai6583e.pdf

Higaona, R; Chauca, L; Zaldivar, M. 1990. Evaluación de los parámetros productivos del cuy criollo. In XII Reunión APPA. Lima: Asociación Peruana de Producción Animal.

Inga, V. 2008. Evaluación de dos niveles de energía digestible y dos niveles de fibra cruda en dietas de crecimiento con exclusión de forraje para cuyes mejorados (Cavia porcellus). Tesis Ing. Zootecnista. Lima, PE. Universidad Nacional Agraria La Molina. 71 p.

INETER (Instituto Nicaragüense de Estudios Territoriales) 2009. Informe estadístico metereológico. Managua, NI. Machii, H. 1989. Varietal differences of nitrogen and amino acid contents in mulberry leaves. Acta Sericologica et entomologica (Japan) 1 , September. 51-61.

Makkar, HPS; Becker, K. 1996. Nutritional value and antinutritional components of whole and ethanol extracted Moringa oleifera leaves. Animal Feed Science Technology 63:211-228.

Mattos, J; Chauca, L; San Martín, F; Carcelén, F; Arbaiza, T. 2003. Uso del ensilado biológico de pescado en la alimentación de cuyes mejorados. Rev Inv Vet Perú 14(2):89-96.

Mc Donald, P; Edwards, RA; Greenhalgh, JFD; Morgan, CA; Sinclair, LA; Wilkinson, RG. 2010. Animal nutrition. 7 ed. $\quad$ Prentice Hall. London, UK. 665 p.

Mercado, L. 1972. Tres niveles de proteínas y dos de energía en raciones para cuyes en crecimiento. Tesis Ing. Zootec nista. Lima, PE. Universidad Nacional Agraria, La Molina. 66 p.

Mendieta Araica, B; Sporndly, E; Reyes Sánchez, N; Sporndly, R. 2011. Feeding Moringa oleifera fresh or ensiled to cows, effects on milk yield and milk flavor. Tropical Animal Health and Production 43:1039-1047. 
Mendieta Araica, B; Sporndly, E; Reyes Sánchez, N; Norell, L; Sporndly, R. 2009. Silage quality when Moringa oleifera is ensiled in mixtures with Elephant grass, sugar cane and molasses. Grass and Forage Science. 64:364-373.

Minitab. 1998. Minitab User's Guide 2. Data Analysis and Quality tools, Release 12 for Windows, Windows 95 and Windows NT. Minitab Inc. Pennsylvania, US.

Miranda A, CI. 2014. Efecto del suministro de nutrientes en la producción de forraje hidropónico de cebada (Hor deum vulgare) y su utilización en la alimentación de cuyes (Cavia porcellus) en crecimiento-engorde. (en línea). Disponible en: http://repositorio.upao. edu.pe/bitstream/upaorep/200/1/MIRANDA_CELITO_SUMINISTRO_NUTRIENTES.pdf

Moreno, A. 1989. Producción de cuyes. Lima, PE. Universidad Nacional Āgraria, La Molina. 132 p.

NRC (National Research Council, US). 1995. Nutrient Requirements of Laboratory Animals: Fourth Revised Edition, Chapter: 4 nutrient requirements of the guinea pig. p 103-124. National Academy Press, 2101 Constitu tion Avenue, N.W. Washington, DC. 20418.

Paredes, L. 1972. Utilización de diferentes niveles de alfalfa en la alimentación de cuyes. Tesis Ing. Zootecnista. Lima, PE. Universidad Nacional Agraria, La Molina. 50 p.

Paucar, J. 1992. Uso de 3 niveles energéticos en suplementos para cuyes destetados y el efecto de la adición de tiro proteína. Tesis de Ing. Zootecnista. Huancayo: Univ Nac del Centro del Perú. 54 p.

Reyes Sánchez, N; Rodríguez, R; Mendieta Araica, B; Mejía Sovalbarro, L; Mora Taylor, A. 2009. Efecto de la suplementación con Moringa oleifera sobre el comportamiento productivo de ovinos alimentados con una dieta basal de pasto guinea (Panicum maximun Jacq.). La Calera 9(13):60-69.

Reyes Sánchez N; Sporndly, E; Ledin I. 2006. Effect of feeding different levels of foliage of Moringa oleifera to creole dairy cows on intake, digestibility, milk production and composition. Livestock Science 101:24-31

Rodríguez Pérez, R; Reyes Sánchez, N; Mendieta Araica, B. 2012. Comportamiento productivo de vacas lecheras alimenta das con Moringa oleifera fresco o ensilado: efecto sobre producción, composición y características organolépticas de leche y queso. La Calera 12(18):45-51.

Sánchez, MD. 1999. Mulberry: an exceptional forage available almost worldwide. World Animal Review 93(2):36- 46.

Saravia, J. 1994. Avances de investigación en la alimentación de cuyes. Lima: INIA/ CIID. Serie guía didáctica. p 17-26.

Shayo, CM. 1997. Uses, yield and nutritive value of mulberry (Morus alba) trees for ruminants in the semi-arid areas of central Tanzania. Tropical Grasslands 31(6):599-604.

Torres, E; Chauca, L; Vergara, V. 2006. Evaluación de dos niveles de energía y proteína en dietas de crecimiento y engor de en cuyes machos. In XXIX Reunión científica anual APPA. Lima: Asociación Peruana de Producción Animal.

Villafranca, M. 2003. Evaluación de tres niveles de fibra en el alimento balanceado para cuyes (Cavia porcellus) en crecimiento y engorde. Tesis de Ingeniero Zootecnista. Universidad Nacional Agraria La Molina, Lima, PE. 90 p. 